\title{
An annotated checklist of vascular plants in and around two major high-altitude wetlands of Lahaul-Spiti, Himachal Pradesh, India
}

\author{
Dipti Dey ${ }^{1}$, Puja Bhojak ${ }^{1}$, K. Chandra Sekar ${ }^{1 *}$, Dhani Arya ${ }^{2}$ \\ 1 Centre for Biodiversity Conservation and Management, G.B. Pant National Institute of Himalayan Environment (NIHE), Kosi-Katarmal, \\ Almora, Uttarakhand, India, • DD: diptiday01@gmail.com (1) https://orcid.org/0000-0003-4586-8842 • PB: pujabhojak007@gmail.com \\ (D) https://orcid.org/0000-0001-5292-4983•KCS: kcsekar1312@rediffmail.com
}

2 Kumaun University, SSJ Campus, Almora, Uttarakhand, India, • DA: dhaniarya@gmail.com

* Corresponding author

\begin{abstract}
We document for the first time the diversity of vascular plants of Chandra Tal and Suraj Tal, two high-altitude wetlands (HAWs) spanning the cold desert region of Lahaul-Spiti, showing the occurrence of 188 species and one variety distributed among 97 genera and 29 families. Only one species, Ephedra intermedia Schrenk \& C.A. Mey. is a gymnosperm, and the other 187 species are angiosperms. Of the angiosperms, Asteraceae is the dominant family, consisting of 27 species and one variety in 17 genera. Among the species, six are classified as threatened, 17 species are native, and two species (Eritrichium nanum (L.) Gaudin and Ranunculus trivedii Aswal \& Mehrotra) are endemic to the Himalayan region. We provide baseline data for future research on the floristic diversity of two major HAWs of Lahaul-Spiti. We also highlight the importance of HAWs for the conservation of species.
\end{abstract}

\section{Keywords}

Chandra Tal, cold desert, floristic diversity, Suraj Tal, Trans-Himalaya

Academic editor: Arjun Prasad Tiwari | Received 26 April 2021 | Accepted 29 November 2021 | Published 16 December 2021

Citation: Dey D, Bhojak P, Chandra Sekar K, Arya D (2021) An annotated checklist of vascular plants in and around two major high-altitude wetlands of Lahaul-Spiti, Himachal Pradesh, India. Check List 17 (6): 1715-1730. https://doi.org/10.15560/17.6.1715

\section{Introduction}

High-altitude wetlands (HAWs) have been defined as "Areas of swamp, marsh, meadow, fen, peat-land or water bodies located at or above an elevation of $2500 \mathrm{~m}$ with an area equal to or greater than five hectares, whether natural or artificial, permanent or temporary, with water that is static or flowing, fresh, brackish or saline" (Anonymous 2010). The Himalayas are home to some of the world's largest and most spectacular glaciers (Brun et al. 2017), and the meltwater from these glaciers and seasonal snow recharges HAWs. These wetlands are an important component in the hydrological regimes of mighty rivers like the Indus, Chandra, and Bhaga, among others.

Geographically, Lahaul-Spiti is the largest district in Himachal Pradesh, covering an area of about $13,883 \mathrm{~km}^{2}$, and part of Indian cold desert in the north-western Himalaya. This region is remarkable for its high-altitude wetlands and lakes which have high biodiversity potential and which are fed by melt water. 
Many studies have been carried out on the floristic diversity (Rau 1960; Nair 1964; Kapahi and Sarin 1979; Koelz 1979; Bhattacharyya and Uniyal 1982; Joshi 2003; Sharma and Samant 2016; Negi et al. 2019) or ethnobotany and resource use in Lahaul-Spiti (Kala 2003, 2006; Samant et al. 2007; Singh et al. 2007; Singh et al. 2009; Rana and Samant 2011). The systematics and taxonomic works in the Lahaul-Spiti region include the publications of Chowdhery and Wadhwa (1984), Aswal and Mehrotra (1994), Murti (2001), Chandra Sekar and Srivastava (2009), and Srivastava and Shukla (2015).

HAWs are one of the most unique as well as the most vulnerable ecosystems in the Himalayans and are very sensitive to global climate change and anthropogenic activities (Schmidt and Psenner 1992). The Space Research Organization recorded 271 HAWs with an area of 575 ha in Himachal Pradesh (Anonymous 2009). These wetlands are important to the people living in this region because they provide livelihood and are considered sacred. But very little information is available about HAWs due to the remoteness and harsh climatic conditions of the region. Any change to the dynamics of these wetlands can have a direct effect on biodiversity. A floristic diversity study is prerequisite and essential in achieving the targets of conservation and management in any ecosystem. Despite enormous biodiversity, very few floristic assessments of high-altitude wetlands in Indian Himalayan Region exist (Rawat and Adhikari 2005; Chandra Sekar and Rawat 2011). Here, we document and assess the diversity of vascular plants of Chandra Tal and Suraj Tal. We also analyse the composition of endemic and threatened elements for their conservation and management.

\section{Study Area}

Our study was carried out in and around Chandra Tal and Suraj Tal, which are in the cold desert region of LahaulSpiti, Himachal Pradesh, India (Fig. 1). Both wetlands are the part of Cold Desert Biosphere Reserve (CDBR), in Indian Trans-Himalaya (Samant et al. 2012). Chandra Tal is located at $32^{\circ} 28.552^{\prime} \mathrm{N}, 077^{\circ} 37.054^{\prime} \mathrm{E}$ at an elevation of about $4300 \mathrm{~m}$; it is about $1750 \mathrm{~m}$ long, $500 \mathrm{~m}$ wide and $5 \mathrm{~km}$ in circumference (Sangewar 2012). It is a tourist destination for trekkers and campers and the source of the Chandra River. Chandra Tal is a wetland of international importance (a Ramsar site) and a part of Chandratal Wildlife Sanctuary which forms the core zone of CDBR (Fig. 2). Due to the presence of nutritive grass, the wetland also is a grazing ground for livestock. Suraj Tal is located at $32^{\circ} 45.749^{\prime} \mathrm{N}, 077^{\circ} 23.957^{\prime} \mathrm{E}$ and is just below the Bara-lacha-la pass at an altitude of about $4800 \mathrm{~m}$. Originating from Suraj Tal is the Bhaga, river which meets the Chandra River downstream at Tandi to form the Chandrabhaga River. We categorized the habitats at our study areas as wet and marshy, moist, dry and rocky, or boulder. The map was produced in ArcGIS v. 10.5 .

\section{Methods}

We conducted extensive and intensive field surveys in and around our two study sites during June to August from 2016 to 2019. Plant specimens were collected and preserved following the methods of Jain and Rao (1977) and housed in the herbarium of the G.B. Pant National Institute of Himalayan Environment, Kosi-Katarmal, Almora (GBP). The collected specimens were identified by comparing them with published floras of Himachal Pradesh (Chowdhery and Wadhwa 1984; Aswal and Mehrotra 1994; Murti 2001; Chandra Sekar and Srivastava 2009) and confirmed with specimens preserved in the Botanical Survey of India, Dehradun (BSD), Central National Herbarium (CAL), and Forest Research Institute, Dehradun (DD). Conservation Assessment and Management Prioritisation (CAMP) and International Union for Conservation of Nature (IUCN), and relevant published literature were used for determining threat status of species (Ved et al. 2003; Mehta et al. 2020; IUCN 2021). We consulted published works for determining endemicity and native status of species in the Indian Himalayan region (Dhar and Samant 1993; Samant et al. 1998; Murti 2001; Singh et al. 2015; Srivastava and Shukla 2015). The habit and habitat of each species were recorded from our field observations, as well as herbarium and literature records. The Angiosperm Phylogeny Group (APG IV) system of classification was followed for arrangement of families (Chase et al. 2016). For nomenclature, Plants of the World Online (POWO) was followed. We provide taxonomic notes and a brief description for each of the threatened species in our list.

\section{Results}

We recorded 188 species and one variety belonging to 97 genera and 29 families (Table 1). Of the 188 species, one (Ephedra intermedia) is a gymnosperm. All other species are angiosperms (Figs. 3-6). Among the recorded threatened species, Aconitum violaceum (IUCN Red List 2021), Rheum spiciforme, and Rhodiola heterodonta are Vulnerable, Arnebia euchroma and Saussurea gossypiphora are Critically Endangered, and Papaver guilelmi-waldemarii is Endangered (Ved et al. 2003).

\section{Threatened species}

Asteraceae

\section{Saussurea gossypiphora D. Don \\ Kasturi Kamal, Snowball Plant}

Material examined. INDIA - Himachal Pradesh - A rare species occurring on alpine slopes, Lahaul-Spiti district, Suraj Tal; $32^{\circ} 45.728^{\prime} \mathrm{N}, 077^{\circ} 23.847^{\prime} \mathrm{E}$; alt. $4624 \mathrm{~m}$; 20.07.2016; DD \& PB 4188, 4190 (GBP).

Identification. Stem 10-30 cm high, covered with long grey or white woolly hairs. Leaves linear, sessile. Heads 


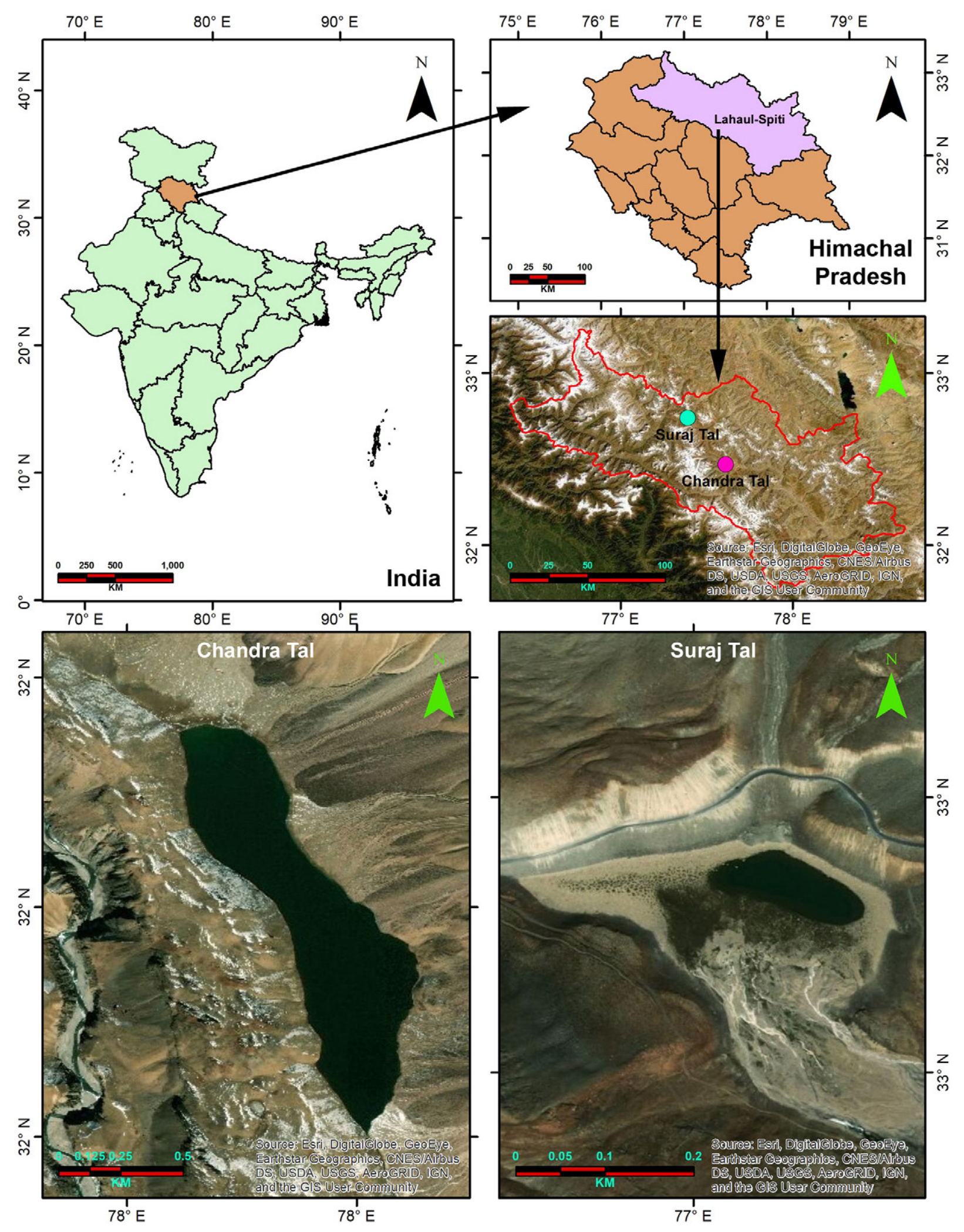

Figure 1. Study area map of two major (Chandra Tal and Suraj Tal) high-altitude wetlands of Lahaul-Spiti. 


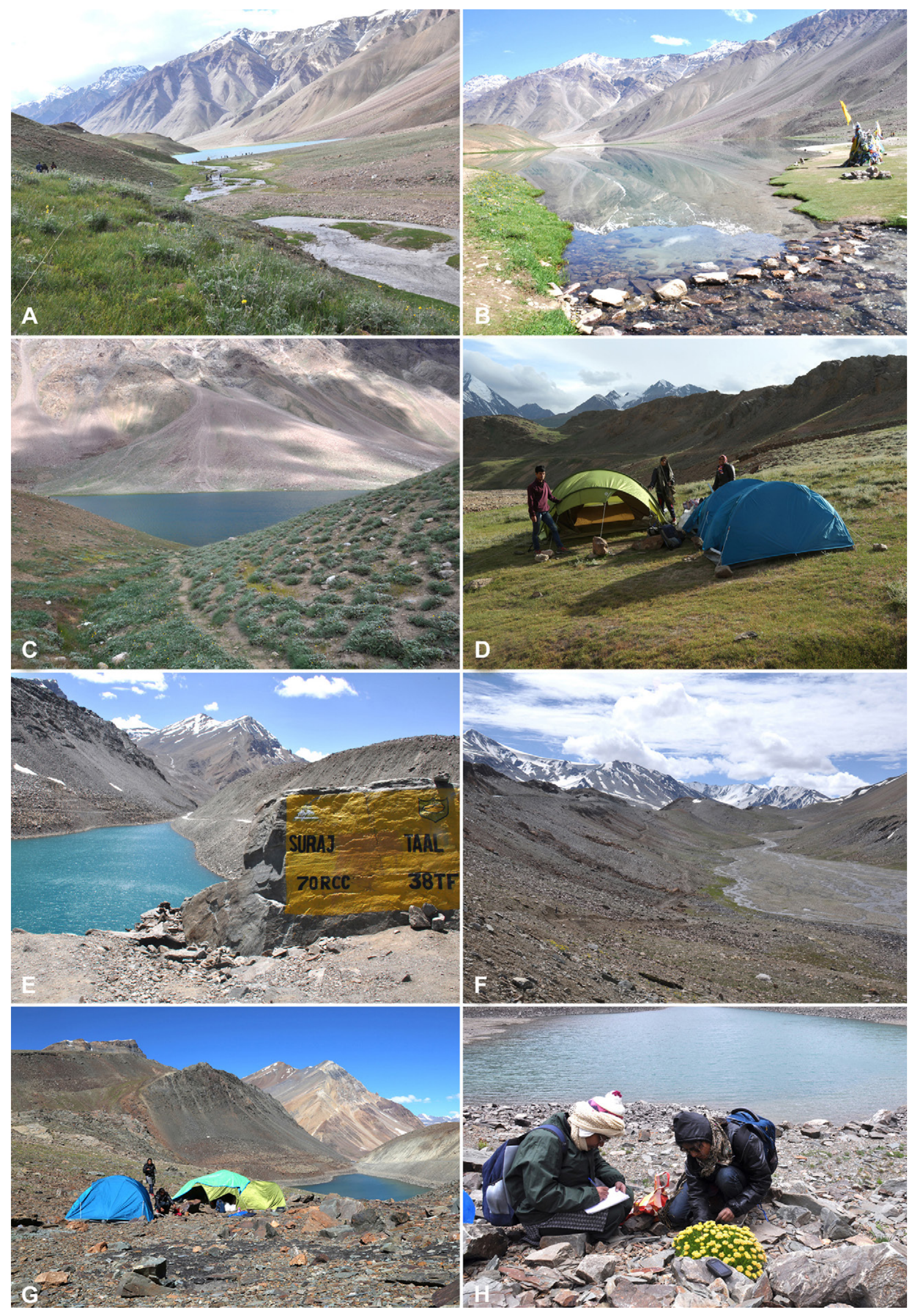

Figure 2. Habitat of Chandra Tal and Suraj Tal high-altitude wetlands of Lahaul-Spiti. A-C. Site view of Chandra Tal. D. Base camp at Chandra Tal. E, F. Site view of Suraj Tal. G. Base camp at Suraj Tal. H. Field survey and collection. 
Table 1. Floristic diversity in and around Chandra Tal and Suraj Tal high-altitude wetlands of Lahaul-Spiti, Himachal Pradesh. Abbreviations: Occurrence: " + " = Present in the valley; Habit: Gr = Grass, H = Herb, Ru = Rush, Sh = Shrub, Se = Sedge; Nativity: ${ }^{* *}=$ Native to Himalayan region; Habitat: DRS = Dry rocky slopes, DR = Dry rocky, SS = Sandy slopes, MOR = Moist rocky, MGS = Moist, grassy stream banks, $\mathrm{RB}=$ River banks, MS = Moist slopes, MSS = Moist sandy slopes, MR = Moist river beds/bank, DGR = Dry grass slopes, DGM = Dry glacial moraines, DS = Dry slopes, DSS = Dry sandy slopes, MM = Moist meadow, MGR = Marshy grass slopes, MGM = Moist glacial moraines, GM = Glacial moraines, MST = Moist stony slopes, $\mathrm{MSH}=$ Moist shady slopes, $\mathrm{DBS}=$ Dry bouldary slopes, $\mathrm{MAS}=$ Marshy slopes, MBS $=$ Moist bouldary slopes, MSW = Marshy stagnant water, DGS = Dry glacial slopes, DST = Dry stony slopes, MME = Moist meadow, MAP = Moist alpine passes, $\mathrm{DRC}=$ Dry rocky crevices, $\mathrm{GM}=$ Grassy meadow, $\mathrm{MG}=$ Moist grassy meadows, $\mathrm{MF}=$ Marshy floating, $\mathrm{MRB}=$ Marshy river beds, MAG = Marshy grassy plains, MRC = Moist rock crevices; Flowering and fruiting: First three letters of the month, Mar $=$ March, Apr $=$ April, May = May, Jun = June, Jul = July, Aug = August, Sep = September, Oct = October; Collection details: DD = Dipti Dey, PB = Puja Bhojak, BSA = B.S. Aswal, SKS = S.K. Srivastava, NLB = N.L. Bor, UCB = U.C. Bhattacharyya, NCN = N.C. Nair, MAR = M.A. Rau; Herbarium: $\mathrm{GBP}=\mathrm{G} . \mathrm{B}$. Pant National Institute of Himalayan Environment, Almora, BSD = Herbarium of Botanical Survey of India, Northern Regional Centre, Dehradun, DD = Forest Research Institute, Dehradun, CAL = Central National Herbarium, Botanical Survey of India, Kolkata, CDRI = Central Drug Research Institute, Lucknow.

\begin{tabular}{|c|c|c|c|c|c|c|c|}
\hline Botanical Name & $\begin{array}{l}\text { Chandra } \\
\text { Tal }\end{array}$ & $\begin{array}{c}\text { Suraj } \\
\text { Tal }\end{array}$ & Habit & Habitat & $\begin{array}{l}\text { Elevation } \\
\quad(\mathrm{m})\end{array}$ & $\begin{array}{c}\text { Flowering \& } \\
\text { fruiting }\end{array}$ & Voucher details \\
\hline \multicolumn{8}{|l|}{ Apiaceae } \\
\hline Bupleurum falcatum L.** (Fig. 3D) & + & & H & DR, SS & $3600-4200$ & Jul-Sept & DD \& PB 3208, 4104, 4165, 4637 (GBP) \\
\hline $\begin{array}{l}\text { Hymenidium stellatum (D.Don) Pimenov \& } \\
\text { Kljuykov (Fig. 5F) }\end{array}$ & & + & H & DRS & $4600-4800$ & Jul-Sept & DD \& PB 4636, 4670 (GBP); BSA 10709 (CDRI) \\
\hline $\begin{array}{l}\text { Pleurospermum candollei (DC.) Benth. ex } \\
\text { C.B.Clarke** (Fig. 5E) }\end{array}$ & & + & H & DRS & $4600-4800$ & Jul-Sept & DD \& PB 3275, 4676, 4685 (GBP); BSA 10980 (CDRI) \\
\hline Vicatia coniifolia DC. & + & & H & MGS & $3600-3800$ & May-Sept & BSA 10499 (CDRI) \\
\hline \multicolumn{8}{|l|}{ Asteraceae } \\
\hline $\begin{array}{l}\text { Ajania tibetica (Hook.f. \& Thomson ex } \\
\text { C.B.Clarke) Tzvelev }\end{array}$ & & + & H & MGM & $4600-4800$ & Jun-Aug & BSA 10736 (CDRI) \\
\hline Allardia glabra Decne.** (Fig. 5l) & & + & H & MS & $4600-4800$ & Jul-Sept & DD \& PB 4654 (GBP); BSA 10728 (CDRI) \\
\hline Allardia nivea Hook.f. \& Thomson ex C.B.Clarke & & + & H & DRS & $4600-4800$ & Aug-Sept & DD \& PB 3282, 4641, 4658 (GBP) \\
\hline Allardia tomentosa Decne. (Fig. 6A) & + & + & H & DSS & $4200-4800$ & Jul-Sept & DD \& PB 3277, 4132 (GBP); BSA 10722, 10731 (CDRI) \\
\hline Anaphalis contorta (D.Don) Hook.f. & & + & H & DRS & $3200-4800$ & Jun-Aug & DD \& PB 3298, 4659 (GBP) \\
\hline Anaphalis nepalensis (Spreng.) Hand.-Mazz. & + & & H & MS & $3400-3600$ & Jun-Sept & BSA 6746 (CDRI); SKS 108312 (BSD)! \\
\hline Anaphalis triplinervis (Sims) C.B.Clarke & + & & H & $\mathrm{DR}, \mathrm{RB}$ & $3200-4200$ & Jul-Sept & DD \& PB 3209 (GBP); SKS 103080, 107087 (BSD)! \\
\hline Aster asterodes Kuntze & & + & H & MR & $4600-4800$ & Jun-Aug & DD \& PB 3289, 4684 (GBP) \\
\hline Aster flaccidus Bunge & + & + & H & MR & $3600-4800$ & Jul-Sept & $\begin{array}{l}\text { DD \& PB 3270, 3280, 4126, } 4663 \text { (GBP); SKS } 105615 \text { (BSD); BSA } 10724 \\
\text { (CDRI) }\end{array}$ \\
\hline Cremanthodium decaisnei C.B.Clarke ${ }^{* *}$ & & + & H & MGM & $4400-4800$ & Jul-Sept & DD \& PB 4668 (GBP); NLB 16359 (DD)! \\
\hline Crepis multicaulis Ledeb. & + & + & H & MOR, SS & $4200-4800$ & Jun-Jul & DD \& PB 3259, 3260, 4631 (GBP); SKS 103091 (BSD)! \\
\hline $\begin{array}{l}\text { Erigeron multiradiatus (Lindl. ex DC.) Benth. } \\
\text { \& Hook.f. }\end{array}$ & + & & H & $M G, R B$ & $3200-3600$ & Jun-Sept & DD \& PB 4189 (GBP); SKS 100319 (BSD)! \\
\hline Inula royleana DC. & & + & H & MR & $3200-4000$ & Jul-Sept & DD \& PB 4192, 4606 (GBP) \\
\hline $\begin{array}{l}\text { Jurinea ceratocarpa var. depressa C.B.Clarke } \\
\text { ex Hook.f. }\end{array}$ & + & & H & MST & $3600-4200$ & Jul-Aug & DD \& PB 3267 (GBP) \\
\hline Lactuca macrorhiza (Royle) Hook.f. & + & & H & MR & $4200-4300$ & Jul-Sept & DD \& PB 4628 (GBP) \\
\hline Leontopodium brachyactis Gand. & + & + & H & MST & $3400-4600$ & Jul-Aug & DD \& PB 4630, 4660 (GBP); UCB 49288 (BSD)! \\
\hline Leontopodium himalayanum DC. & + & & H & MR & $4200-4300$ & Jul-0ct & DD \& PB 4113, 4155, 4173 (GBP); SKS 103039 (BSD)!; BSA 6966 (CDRI) \\
\hline $\begin{array}{l}\text { Leontopodium nanum (Hook.f. \& Thomson ex } \\
\text { C.B.Clarke) Hand.-Mazz. }\end{array}$ & + & + & H & DGR & $3600-4800$ & Jul-0ct & DD \& PB 3297 (GBP); SKS 100368 (BSD)!, NCN 16763 (BSD)! \\
\hline Neobrachyactis roylei (DC.) Brouillet & + & & H & MR & $4250-4300$ & Jul-Sept & DD \& PB 3252 (GBP); SKS 98997 (BSD)! \\
\hline $\begin{array}{l}\text { Psychrogeton poncinsii (Franch.) Y.Ling \& } \\
\text { Y.L.Chen (Fig. 5H) }\end{array}$ & + & + & H & $\mathrm{MOR}, \mathrm{RB}$ & $4200-4800$ & Jun-Sept & DD \& PB 3243, 4139, 4172 (GBP); BSA 10974 (CDRI) \\
\hline Richteria pyrethroides Kar. \& Kir.(Fig. 4J) & + & & H & DST & $3300-4800$ & Jun-Aug & DD \& PB 4133 (GBP) \\
\hline Saussurea glacialis Herder (Fig. 5L) & & + & H & MST & $3800-4800$ & Jul-Aug & DD \& PB 3281, 4632 (GBP) \\
\hline Saussurea gnaphalodes (Royle ex DC.) & & + & H & MGM & $4600-4800$ & Jul-Aug & BSA 10707 (CDRI) \\
\hline Saussurea gossypiphora D. Don.** & & + & H & MST & $4300-5000$ & Jul-Sept & DD \& PB 4188, 4190 (GBP) \\
\hline $\begin{array}{l}\text { Sericocarpus asteroides (L.) Britton, Sterns \& } \\
\text { Poggenb.** }\end{array}$ & + & & H & MSS & $4250-4300$ & Jul-Sept & DD \& PB 3210, 3251, 4115 (GBP), SKS 100840 (BSD)! \\
\hline Taraxacum eriopodum DC. & + & & H & MS & $3200-3800$ & Jun-Sept & SKS 103053 (BSD)! \\
\hline Taraxacum leucanthum (Ledeb.) Ledeb. & + & & H & MR & $4250-4300$ & Jul-Sept & SKS 103038 (BSD)! \\
\hline Taraxacum officinale F.H.Wigg. (Fig. 4L) & + & & $\mathrm{H}$ & MR & $3200-3800$ & Jun-Sept & DD \& PB 3230, 4140 (GBP); SKS 100381 (BSD)!; NCN 16767 (BSD)! \\
\hline \multicolumn{8}{|l|}{ Boraginaceae } \\
\hline $\begin{array}{l}\text { Arnebia euchroma (Royle ex Benth.) } \\
\text { I.M.Johnst.** (Fig. 3B) }\end{array}$ & + & & H & DST & $3500-4000$ & Jun-Jul & $D D \& P B 4136$ (GBP) \\
\hline Eritrichium nanum (L.) Gaudin & + & & H & MS & $3400-4000$ & Jun-Aug & BSA 10238 (CDRI); SKS 103037A (BSD)! \\
\hline Eritrichium spathulatum (Benth.) C.B.Clarke & & + & H & MGM & $4600-4800$ & Jul-Aug & NLB 8671 (DD)! \\
\hline Eritrichium villosum (Ledeb.) Bunge & & + & H & DST & $4000-4800$ & Jun-Aug & DD \& PB 3295, 4673, 4683 (GBP); NLB 15448 (DD)! \\
\hline Myosotis sylvatica Ehrh. ex Hoffm. (Fig. 3H) & + & & H & DST & $3200-4000$ & Jun-Aug & SKS $103037 B(B S D) !$ \\
\hline
\end{tabular}




\begin{tabular}{|c|c|c|c|c|c|c|c|}
\hline Botanical Name & $\begin{array}{l}\text { Chandra } \\
\text { Tal }\end{array}$ & $\begin{array}{l}\text { Suraj } \\
\text { Tal }\end{array}$ & Habit & Habitat & $\begin{array}{l}\text { Elevation } \\
\text { (m) }\end{array}$ & $\begin{array}{c}\text { Flowering \& } \\
\text { fruiting }\end{array}$ & Voucher details \\
\hline Myosotis verna Nutt. & + & & $\mathrm{H}$ & MRC & $3200-3600$ & May-Jun & DD \& PB 4175, 4176 (GBP) \\
\hline \multicolumn{8}{|l|}{ Brassicaceae } \\
\hline $\begin{array}{l}\text { Aphragmus oxycarpus (Hook.f. \& Thomson) } \\
\text { Jafri (Fig. 4A) }\end{array}$ & & + & H & DRC & $3600-4800$ & Jun-Jul & NLB 15221 (DD)! \\
\hline Arabis amplexicaulis Edgew. & + & & H & MS & $3200-4000$ & Jun-Sept & SKS 103071 (BSD)! \\
\hline Arabis recta Vill. & + & & H & MM & $3600-4000$ & Jun-Sept & DD \& PB 3255, 4108 (GBP); SKS 103049 (BSD)! \\
\hline Braya rosea Bunge & & + & H & DGM & $4600-4800$ & Jun-Aug & NLB 213 (DD)! \\
\hline Chorispora sabulosa Cambess. (Fig. 4B) & & + & H & MGM & $3800-4800$ & Jun-Aug & DD \& PB 3291, 4646 (GBP) \\
\hline $\begin{array}{l}\text { Crucihimalaya tibetica (Hook.f. \& Thomson) Al- } \\
\text { Shehbaz, D.A.German \& M.Koch }\end{array}$ & + & & H & MS & $3200-4000$ & Jun-Sept & SKS 103069 (BSD)! \\
\hline $\begin{array}{l}\text { Crucihimalaya wallichii (Hook.f. \& Thomson) Al- } \\
\text { Shehbaz, 0'Kane \& R.A.Price }\end{array}$ & + & & H & MGR & $4200-4300$ & Apr-Aug & DD \& PB 4129 (GBP) \\
\hline Dilophia salsa Thomson & & + & H & MGM & $4600-4800$ & Jun-Jul & BSA 11005 (CDRI) \\
\hline Draba altaica (C.A. Mey.) Bunge & & + & H & DST & $4600-4800$ & Jun-Aug & BSA 10723 (CDRI); SKS 103103 (BSD)! \\
\hline Drabaglomerata Royle & + & + & H & MST & $3800-4800$ & Jun-Aug & DD \& PB 2557 (GBP); SKS 103029 (BSD)!, UCB 40839 (BSD)! \\
\hline Draba lanceolata Royle & + & & H & MST & $3200-3800$ & Jun-Aug & SKS 100380 (BSD)! \\
\hline Draba oreades Schrenk (Fig. 5C) & & + & H & MST & $4600-4800$ & Jun-Aug & DD \& PB 4639 (GBP) \\
\hline Draba setosa Royle & & + & H & DST, GM & $4600-4800$ & May-Aug & DD \& PB 4661, 4680 (GBP); BSA 10720 (CDRI) \\
\hline Sisymbrium orientale L. & & + & H & MOR & $4000-4600$ & Apr-Aug & DD \& PB 4682 (GBP) \\
\hline \multicolumn{8}{|l|}{ Campanulaceae } \\
\hline Cyananthus lobatus Wall. ex Benth. & + & & H & MS & $3300-4500$ & Jul-Sept & DD \& PB 4629 (GBP) \\
\hline \multicolumn{8}{|l|}{ Caprifoliaceae } \\
\hline $\begin{array}{l}\text { Lonicera spinosa (Decne.) Jacq. ex Walp. } \\
\text { (Fig. 6H) }\end{array}$ & + & + & Sh & DST & $3600-4600$ & Jun-Jul & DD \& PB 4153 (GBP); NLB 8698 (DD)! \\
\hline \multicolumn{8}{|l|}{ Caryophyllaceae } \\
\hline Cerastium glomeratum Thuill. & & + & H & MS & $3800-4200$ & Jun-Sept & DD \& PB 2559 (GBP) \\
\hline Cherleria biflora (L.) A.J.Moore \& Dillenb. & & + & H & MST & $4600-4800$ & Jul-Sept & BSA 10297 (CDRI) \\
\hline Dichodon cerastoides (L.) Rchb.(Fig. 5A) & + & + & H & MR & $4200-4800$ & May-Aug & DD \& PB 4645 (GBP); UCB 40826 (BSD)! \\
\hline $\begin{array}{l}\text { Eremogone kansuensis (Maxim.) Dillenb. \& } \\
\text { Kadereit }\end{array}$ & + & & H & MR & $3600-4000$ & Jun-Jul & DD \& PB 4122 (GBP) \\
\hline Silene gonosperma (Rupr.) Bocquet & + & + & H & MSS & $3200-4400$ & Jun-Jul & DD \& PB 4687 (GBP); SKS 100397 (BSD)! \\
\hline Silene graminifolia 0tth & + & & H & DST & $4250-4300$ & Jun-Jul & DD \& PB 3244, 4603 (GBP) \\
\hline Silenenepalensis Majumdar & & + & H & MSH & $3400-4200$ & Jul-0ct & DD \& PB 4652 (GBP) \\
\hline $\begin{array}{l}\text { Silene songarica (Fisch., C.A. Mey. \& Avé-Lall.) } \\
\text { Bocquet }\end{array}$ & & + & H & DBS & $3200-4600$ & Jun-Sept & DD \& PB 3287 (GBP) \\
\hline Silene uralensis (Rupr.) Bocquet & + & & H & MS & $3800-4200$ & Jun-Jul & UCB 49453 (BSD)! \\
\hline Stellaria decumbens Edgew. & + & + & H & MSS & $3400-4200$ & Jun-Sept & DD \& PB 3274, 4120, 4124, 4657 (GBP); SKS 107131 (BSD)! \\
\hline Stellaria longifolia Muhl. ex Willd. & + & & H & MSS & $3200-3400$ & May-Jul & $D D \& P B 3237$ GBP \\
\hline Stellaria williamsiana Kozhevn. & + & & H & MSS & $3200-3800$ & Jul-Aug & DD \& PB 3224, 4107, 4128 (GBP) \\
\hline $\begin{array}{l}\text { Thylacospermum caespitosum (Cambess.) } \\
\text { Schischk. }\end{array}$ & & + & H & DBS & $4400-4800$ & Jun-Sept & DD \& PB 4626 (GBP); BSA 10706 (CDRI) \\
\hline \multicolumn{8}{|l|}{ Crassulaceae } \\
\hline Hylotelephium ewersii (Ledeb.) H.Ohba** & & + & H & MBS & $3600-4500$ & Jul-Sept & DD \& PB 4649, 4679, 4681 (GBP) \\
\hline $\begin{array}{l}\text { Rhodiola crenulata (Hook. f. \& Thomson) H. } \\
\text { Ohba }\end{array}$ & + & + & H & MS, MRC & $3600-4800$ & Jun-Sept & DD \& PB 3245, 4166, 4199, 4643, 4672, 4888 (GBP); BSA 10717 (CDRI) \\
\hline $\begin{array}{l}\text { Rhodiola heterodonta (Hook. f. \& Thomson) } \\
\text { Boriss. }\end{array}$ & & + & H & DRS & $3300-5000$ & Jun-Aug & DD \& PB 4620 (GBP) \\
\hline Rhodiola himalensis (D. Don) S.H. Fu (Fig. 5J) & + & & H & MR & $4250-4300$ & Jun-Aug & DD \& PB 4121, 4198 (GBP) \\
\hline Rhodiola imbricata Edgew. (Fig. 5K) & & + & H & DST & $4200-4800$ & Jun-Aug & DD \& PB 3276, 4651 (GBP) \\
\hline Rosularia alpestris (Kar. \& Kir.) Boriss. (Fig. 6M) & + & & H & MST & $3200-3800$ & Jun-Jul & DD \& PB 3217, 4168 (GBP) \\
\hline \multicolumn{8}{|l|}{ Cyperaceae } \\
\hline Carex borii Nelmes & & + & Se & DGS & $3800-4900$ & Jun-Aug & BSA 11001 (CDRI) \\
\hline Carex infuscata Nees & & + & Se & MR & $4600-4800$ & Jul-Sept & BSA 10292 (CDRI) \\
\hline Carex maritima Gunnerus (Fig. 6C) & & + & Se & MR & $4600-4800$ & Jun-Jul & DD \& PB 2558B (GBP) \\
\hline Carex melanantha C.A. Mey. & + & & Se & MSS & $4200-4400$ & Jul-Aug & DD \& PB 3266, 4184 (GBP), SKS 107111 (BSD)! \\
\hline Carex micropoda C.A.Mey. (Fig. 6D) & + & + & Se & MSS & $4200-4800$ & Jul-Aug & DD \& PB 3288, 4623 (GBP), BSA 10973 (CDRI) \\
\hline Carex nudicarpa (Y.C.Yang) S.R.Zhang & & + & Se & MSS & $4600-4800$ & Jul-Aug & UCB 40828 (BSD)! \\
\hline Carex ovoidispica 0.Yano & & + & Se & MSS & $4600-4800$ & Jul-Aug & NLB 171 (DD)! \\
\hline Carexparvula 0.Yano & & + & Se & MSS & $4600-4800$ & Jul-Aug & BSA 10998 (CDRI) \\
\hline Carex pamirensis C.B. Clarke & + & & Se & MSH & $4200-4400$ & Jul-Sept & DD \& PB 4697 (GBP) \\
\hline Eleocharis palustris (L.) Roem. \& Schult. & + & + & Se & MRB & $4200-4800$ & May-Sept & DD \& PB 3211 (GBP) \\
\hline \multicolumn{8}{|l|}{ Ephedraceae } \\
\hline $\begin{array}{l}\text { Ephedra intermedia Schrenk \& C.A.Mey. } \\
\text { (Fig. 4E) }\end{array}$ & + & + & Sh & DRC & $3600-4800$ & Jul-0ct & DD \& PB 3242, 4148, 4194, 4648, 4692(GBP) \\
\hline
\end{tabular}




\begin{tabular}{|c|c|c|c|c|c|c|c|}
\hline Botanical Name & $\begin{array}{l}\text { Chandra } \\
\text { Tal }\end{array}$ & $\begin{array}{c}\text { Suraj } \\
\text { Tal }\end{array}$ & Habit & Habitat & $\begin{array}{c}\text { Elevation } \\
\text { (m) }\end{array}$ & $\begin{array}{l}\text { Flowering \& } \\
\text { fruiting }\end{array}$ & Voucher details \\
\hline \multicolumn{8}{|l|}{ Fabaceae } \\
\hline Astragalus cariensis Boiss. (Fig. 6B) & + & & $\mathrm{H}$ & $D S, R B$ & $3600-4200$ & Jun-Aug & DD \& PB 3265, 4111 (GBP); SKS 103092, 103093 (BSD)! \\
\hline Astragalus himalayanus Klotzsch & + & & H & MR & $3200-3800$ & Jun-Sept & $D D \& P B 3263 B(G B P)$ \\
\hline Astragalus strictus Benth. & + & & $\mathrm{H}$ & MSH, MST & $3200-3600$ & Jul-Sept & DD \& PB 3221, 4112 (GBP); NCN 21073 (BSD)! \\
\hline Cicer microphyllum Royle ex Benth. & + & & $\mathrm{H}$ & DSS & $3200-4000$ & Jun-Aug & DD\& PB 3253, 4131 (GBP); SKS 107096 (BSD)! \\
\hline Oxytropis humifusa Kar. \& Kir. & + & & H & MS & $3200-4000$ & Jul-Sept & SKS 103034 (BSD)! \\
\hline Oxytropis lapponica (Wahlenb.) J.Gay & + & + & $\mathrm{H}$ & $M S, R B, D R$ & $3200-4800$ & Jun-Aug & DD \& PB 3214, 4106, 4149, 4178, 4696 (GBP); SKS 100391 (BSD)! \\
\hline Oxytropis microphylla (Pall.) DC. & & + & H & MGM & $4600-4800$ & Jun-Aug & NLB 8659 (DD)! \\
\hline Oxytropis mollis Royle ex Benth. & + & + & $\mathrm{H}$ & MSS & $4200-4800$ & May-Sept & DD \& PB 3263A, 3296 (GBP); SKS 103031, 103075 (BSD)! \\
\hline Trigonella emodi Benth. & + & & $\mathrm{H}$ & MS & $3200-3600$ & Jun-Sept & DD \& PB 3233 (GBP) \\
\hline \multicolumn{8}{|l|}{ Gentianaceae } \\
\hline Comastoma tenellum (Rottb.) Toyok. & + & & $\mathrm{H}$ & MRB & $3600-4000$ & Jul-Aug & DD \& PB $3257 A$ (GBP) \\
\hline $\begin{array}{l}\text { Gentiana argentea (Royle ex D.Don) Royle ex } \\
\text { D.Don }\end{array}$ & + & & H & MME & $3600-4400$ & Apr-Jul & SKS 103046, 107114 (BSD)! \\
\hline Gentiana coronata Royle & + & & H & MR & $4250-4300$ & Jun-Aug & DD \& PB 3247 (GBP) \\
\hline Gentiana leucomelaena Maxim. & + & + & H & MS & $3400-4800$ & May-Aug & DD \& PB 3220A (GBP); BSA 10291 (CDRI) \\
\hline Gentiana nivalis L. (Fig. 6E) & + & & $\mathrm{H}$ & MAG & $3200-3800$ & Aug-0ct & DD \& PB 3220B, 4174 (GBP) \\
\hline Gentiana nubigena Edgew. & & + & $\mathrm{H}$ & MME & $4600-4800$ & Jul-Aug & UCB 52337 (BSD)! \\
\hline Gentiana phyllocalyx C.B. Clarke & + & & $\mathrm{H}$ & MR & $4200-4300$ & Jun-0ct & DD \& PB 4193 (GBP) \\
\hline Gentianella aurea (L.) Harry Sm. & + & & $\mathrm{H}$ & MG & $4200-4600$ & Jun-Aug & BSA 6671 (CDRI) \\
\hline Gentiana membranulifera T.N.Ho & + & & $\mathrm{H}$ & MR & $4200-4300$ & May-Aug & DD \& PB 3234, 4144 (GBP) \\
\hline $\begin{array}{l}\text { Gentianella moorcroftiana (Wall. ex Griseb.) } \\
\text { Airy Shaw }\end{array}$ & + & & $\mathrm{H}$ & MME & $3200-4200$ & Jul-Aug & UCB 49447 (BSD)! \\
\hline Gentianopsis detonsa (Rottb.) Ma** & + & & $\mathrm{H}$ & MR & $4200-4300$ & Jul-0ct & DD \& PB 3226, 4118 (GBP) \\
\hline Gentianopsis paludosa (Hook.f.) Ma & + & & $\mathrm{H}$ & MR & $3800-4400$ & Jul-Aug & UCB 52210 (BSD)! \\
\hline Lomatogonium carinthiacum (Wulfen) A.Braun & + & & $\mathrm{H}$ & MR & $4200-4300$ & Jul-0ct & DD \& PB 4186 (GBP); SKS 100388 (BSD)! \\
\hline \multicolumn{8}{|l|}{ Geraniaceae } \\
\hline Geranium pratense $\mathrm{L}$. & + & & H & DS & $3200-3800$ & Jul-Aug & DD \& PB 3249 (GBP) \\
\hline $\begin{array}{l}\text { Geranium wallichianum D. Don ex Sweet** } \\
\text { (Fig. 3E) }\end{array}$ & + & & $\mathrm{H}$ & MST & $3200-3800$ & Jun-Sept & SKS 100309 (BSD)! \\
\hline \multicolumn{8}{|l|}{ Juncaceae } \\
\hline Juncus allioides Franch. (Fig. 6F) & + & & Ru & MR & $4250-4300$ & Jul-Aug & DD \& PB 3246 (GBP) \\
\hline Juncus himalensis Klotzsch (Fig. 6G) & + & & Ru & MR & $4200-4300$ & Jun-Aug & DD \& PB 4611 (GBP) \\
\hline Juncus leucomelas Royle ex D. Don & + & & Ru & MR & $4200-4300$ & Jul-Aug & DD \& PB 3240 (GBP) \\
\hline \multicolumn{8}{|l|}{ Juncaginaceae } \\
\hline Triglochin maritima L. & + & & $\mathrm{H}$ & MAS & $3200-4600$ & May-0ct & DD \& PB 3213, 4102, 4146, 4147 (GBP), SKS 108319 (BSD)! \\
\hline \multicolumn{8}{|l|}{ Lamiaceae } \\
\hline Elsholtzia eriostachya (Benth.) Benth. (Fig. 4D) & + & & H & MS & $3200-4600$ & Jul-Sept & DD \& PB 3225, 4125, 4152, 4617 (GBP) \\
\hline Nepeta eriostachya Benth.** & + & + & H & MS & $3200-4800$ & Jul-Sept & DD \& PB 3223, 4162, 4635 (GBP); SKS 108307 (BSD)! \\
\hline Nepeta longibracteata Benth. (Fig. 5D) & & + & H & DGS & $4400-4800$ & Jul-Aug & DD \& PB 3284, 4642, 4694 (GBP); BSA 10725 (CDRI) \\
\hline Thymus linearis Benth. (Fig. 4K) & + & & H & MSS & $3200-4000$ & Jul-Sept & DD \& PB 3241, 4156, 4160, 4177 (GBP); SKS 103079 (BSD)!; BSA 8821 (CDRI) \\
\hline \multicolumn{8}{|l|}{ Liliaceae } \\
\hline Gagea gageoides (Zucc.) Vved. & & + & H & DBS & $4600-4800$ & Jul-Aug & BSA 10327 (CDRI) \\
\hline Gagea serotina (L.) Ker Gawl. (Fig. 4G) & & + & $\mathrm{H}$ & MST & $4600-4800$ & Jun-Aug & DD \& PB 4677, 4689 (GBP) \\
\hline \multicolumn{8}{|l|}{ Onagraceae } \\
\hline Epilobium latifolium L. & & + & $\mathrm{H}$ & DST & $4000-4800$ & Jul-Sept & DD \& PB 3290 (GBP) \\
\hline Epilobium laxum Royle & + & & $\mathrm{H}$ & MR & $3200-4200$ & Jul-Sept & DD \& PB 3231 (GBP) \\
\hline Epilobium leiophyllum Hausskn. & & + & $\mathrm{H}$ & MGM & $4600-4800$ & Jul-Aug & BSA 10733 (CDRI) \\
\hline Epilobium palustre $\mathrm{L}$. & + & & H & $M S, R B$ & $3200-3800$ & Jun-Sept & DD \& PB 4110 (GBP) \\
\hline Epilobium royleanum Hausskn. (Fig. 4F) & + & & $\mathrm{H}$ & MGM & $4200-4600$ & Jun-Aug & SKS 100386 (BSD)! \\
\hline \multicolumn{8}{|l|}{ Orobanchaceae } \\
\hline Euphrasia himalayica Wettst. & + & & $\mathrm{H}$ & MS & $3600-4200$ & May-Jul & DD \& PB 3261 (GBP) \\
\hline Pedicularis hoffmeisteri Klotzsch & + & & $\mathrm{H}$ & MS & $4200-4300$ & Jun-Aug & DD \& PB 4130, 4195 (GBP) \\
\hline Pedicularis longiflora Rudolph (Fig. 6J) & + & & H & MR & $3200-4200$ & Jun-Aug & DD \& PB 4616 (GBP) \\
\hline Pedicularis pectinata Wall. ex Benn. & + & & H & MR & $3200-3600$ & Jun-Aug & DD \& PB 4145, 4163 (GBP); SKS 108309 (BSD) \\
\hline Pedicularis rhinanthoides Schrenk (Fig. 6K) & + & & H & MR & $3200-4200$ & Jul-Sept & DD \& PB 3238, 3248 (GBP) \\
\hline Pedicularis roylei Maxim. & & + & $\mathrm{H}$ & MGM & $4200-4850$ & Jul-Aug & DD \& PB 4669, 4693 (GBP), UCB 52339 (BSD)!; BSA 10982 (CDRI) \\
\hline \multicolumn{8}{|l|}{ Papaveraceae } \\
\hline Corydalis crassifolia Royle & & + & H & MGM & $4600-4800$ & Jul-Aug & NLB 13983 (DD)! \\
\hline Corydalis govaniana Wall.** (Fig. 5B) & & + & $\mathrm{H}$ & DST & $3400-4800$ & May-Aug & DD \& PB 3283 (GBP) \\
\hline Corydalis meifolia Wall.** & + & + & $\mathrm{H}$ & MST & $3600-4800$ & Jun-Aug & DD \& PB 3257B, 3286, 4665 (GBP); BSA 10995 (CDRI) \\
\hline Corydalis moorcroftiana Wall. ex Hook. f. \& & & + & $\mathrm{H}$ & MST & $4000-4800$ & Jul-Aug & DD \& PB 4678, 4686 (GBP) \\
\hline
\end{tabular}




\begin{tabular}{|c|c|c|c|c|c|c|c|}
\hline Botanical Name & $\begin{array}{l}\text { Chandra } \\
\text { Tal }\end{array}$ & $\begin{array}{l}\text { Suraj } \\
\text { Tal }\end{array}$ & Habit & Habitat & $\begin{array}{l}\text { Elevation } \\
\quad(\mathrm{m})\end{array}$ & $\begin{array}{l}\text { Flowering \& } \\
\text { fruiting }\end{array}$ & Voucher details \\
\hline $\begin{array}{l}\text { Papaver guilelmi-waldemarii (Klotzsch) Chris- } \\
\text { tenh. \& Byng (Fig. 3G) }\end{array}$ & & + & $\mathrm{H}$ & DRC & $3400-4800$ & Jul-0ct & MAR 5913 (BSD)! \\
\hline \multicolumn{8}{|l|}{ Plantaginaceae } \\
\hline Hippuris vulgaris L. (Fig. 3F) & + & & $\mathrm{H}$ & MAS & $3200-3800$ & Jun-Jul & DD \& PB 4151 (GBP) \\
\hline Veronica beccabunga L. (Fig. 3L) & + & & $\mathrm{H}$ & MR & $3200-3600$ & Jun-Sept & DD \& PB 3227,4119, 4157 (GBP); SKS 103113 (BSD)! \\
\hline Veronica biloba Schreb. ex L. & + & & $\mathrm{H}$ & MR & $3200-3600$ & Jul-Sept & DD \& PB 4103, 4164 (GBP) \\
\hline \multicolumn{8}{|l|}{ Poaceae } \\
\hline Agrostis castellana Boiss. \& Reut. & + & & $\mathrm{Gr}$ & MS & $3800-4400$ & Jul-Sept & DD \& PB 4159, 4610 (GBP) \\
\hline Elymus dahuricus Turcz. ex Griseb. & & + & $\mathrm{Gr}$ & MS & $4600-4800$ & Jul-Aug & NLB 326 (DD)! \\
\hline Festuca valesiaca Schleich. ex Gaudin & & + & $\mathrm{Gr}$ & MS & $4600-4800$ & Jun-Aug & NLB 15070 (DD)! \\
\hline Melica persica Kunth & + & + & $\mathrm{Gr}$ & DS & $4200-4800$ & May-Aug & DD \& PB 4601, 4602 (GBP) \\
\hline Phleum alpinum $\mathrm{L}$. & + & & Gr & MS & $3200-4000$ & Jul-Sept & SKS 100333 (BSD)! \\
\hline Poaannua L. & & + & $\mathrm{Gr}$ & MRC & $4600-4800$ & Jun-Jul & NCN 16496 (BSD)! \\
\hline Poa alpina L. & + & & $\mathrm{Gr}$ & GM & $4200-4300$ & Jul-Aug & SKS 103104, 103114 (BSD)! \\
\hline Poaglauca Vahl & & + & $\mathrm{Gr}$ & MAP & $4600-4800$ & Jun-Jul & NLB 13972 (DD) \\
\hline Poa persica Trin. & & + & $\mathrm{Gr}$ & MRC & $4600-4800$ & Jun-Jul & NLB 12122 (DD)! \\
\hline Poa supina Schrad. & + & & Gr & GM & $4200-4300$ & Jun-Jul & UCB 48763 (BSD)!, NCN 17065 (BSD)! \\
\hline Puccinellia kashmiriana Bor & & + & $\mathrm{Gr}$ & MAP & $4600-4800$ & Jul-Aug & NLB 16360 (DD) \\
\hline Trisetum spicatum (L.) K.Richt. & + & & $\mathrm{Gr}$ & GM & $4200-4300$ & Jul-Aug & UCB 45071(BSD)! \\
\hline \multicolumn{8}{|l|}{ Polygonaceae } \\
\hline Bistorta affinis (D.Don) Greene & + & + & $\mathrm{H}$ & MG & $4200-4800$ & Jun-Sept & DD \& PB 3207, 4197, 4619 (GBP); SKS 100369, 103056, (BSD)! \\
\hline Bistorta vivipara (L.) Delarbre & + & & $\mathrm{H}$ & MG & $3200-4800$ & Jun-Aug & DD \& PB 3215, 4621 (GBP); SKS 100376 (BSD)! \\
\hline Oxyria digyna (L.) Hill (Fig. 6l) & + & + & $\mathrm{H}$ & DR & $3200-4800$ & Jun-Sept & DD \& PB 3258 (GBP); SKS 103078, 103109 (BSD)! \\
\hline Polygonum aviculare L. & + & & $\mathrm{H}$ & MG & $3200-3800$ & Jul-Sept & DD \& PB 4171 (GBP) \\
\hline Polygonum cognatum Meisn. (Fig. 5G) & + & + & $\mathrm{H}$ & MG & $3200-3800$ & Jul-Sept & $\begin{array}{l}\text { DD \& PB 3228, 4117, 4634, } 4664 \text { (GBP); SKS 103085, 106265, } 107148 \\
\text { (BSD)! }\end{array}$ \\
\hline Polygonum paronychioides C.A. Mey. & + & + & H & DR & $3200-3800$ & Jun-Sept & DD \& PB 3273, 4154, 4653 (GBP) \\
\hline Polygonum recumbens Royle ex Bab. & + & & $\mathrm{H}$ & MS & $3200-3800$ & Jun-Aug & SKS 108321 (BSD)! \\
\hline Polygonum rottboellioides Jaub. \& Spach & + & & $\mathrm{H}$ & MS & $3200-3800$ & Jun-Sept & SKS 107149 (BSD)! \\
\hline Rheum spiciforme Royle** (Fig. 3J) & + & + & $\mathrm{H}$ & MSS & $3200-4800$ & Jun-Aug & DD \& PB 3735, 4622 (GBP); BSA 10967 (CDRI) \\
\hline \multicolumn{8}{|l|}{ Potamogetonaceae } \\
\hline Potamogeton crispus L. & + & + & $\mathrm{H}$ & MF & $3200-4200$ & May-Aug & SKS 100398 (BSD)! \\
\hline Potamogeton natans $\mathrm{L}$. & + & & $\mathrm{H}$ & MF & $3200-4200$ & May-Aug & $D D \& P B 4116$ (GBP) \\
\hline Potamogeton nodosus Poir. (Fig. 6L) & + & & $\mathrm{H}$ & MSW & $3200-4200$ & May-Aug & DD \& PB 4150 (GBP) \\
\hline Stuckenia pectinata (L.) Börner (Fig. 3K) & + & & $\mathrm{H}$ & MSW & $4200-4400$ & May-0ct & DD \& PB 3254, 4123, 4618 (GBP) \\
\hline \multicolumn{8}{|l|}{ Primulaceae } \\
\hline Androsace sempervivoides Jacquem. ex Duby & + & & $\mathrm{H}$ & MS & $3600-4800$ & Jun-Aug & DD \& PB 4141 (GBP) \\
\hline Primula minutissima Jacquem. ex Duby (Fig. 4I) & + & + & $\mathrm{H}$ & MS & $3400-4600$ & Jun-Jul & DD \& PB 4655 (GBP) \\
\hline \multicolumn{8}{|l|}{ Ranunculaceae } \\
\hline Aconitum rotundifolium Kar. \& Kir. & & + & $\mathrm{H}$ & MST & $4600-4800$ & Jul-Sept & NLB 224 (DD)! \\
\hline $\begin{array}{l}\text { Aconitum violaceum Jacquem. ex Stapf** } \\
\text { (Fig. } 3 \text { A) }\end{array}$ & + & & $\mathrm{H}$ & MS & $3400-4800$ & Jul-Sept & NCN 16764 (BSD)! \\
\hline Delphinium brunonianum Royle** (Fig. 4C) & & + & $\mathrm{H}$ & DST & $4200-4800$ & Jul-Sept & DD \& PB 3285, 4612 (GBP); BSA 10721 (CDRI) \\
\hline $\begin{array}{l}\text { Eriocapitella rupicola (Cambess.) Christenh. } \\
\text { \& Byng }\end{array}$ & & + & $\mathrm{H}$ & MGM & $4600-4800$ & Jul-Sept & BSA 10296 (CDRI) \\
\hline Halerpestes tricuspis (Maxim.) Hand.-Mazz. & + & + & $\mathrm{H}$ & MAS & $3200-4800$ & Jun-Jul & DD \& PB 3216, 3229, 4109, 4143, 4691 (GBP); SKS 103059, 103102 (BSD)! \\
\hline $\begin{array}{l}\text { Oxygraphis endlicheri (Walp.) Bennet \& Sum. } \\
\text { Chandra }\end{array}$ & & + & $\mathrm{H}$ & MS & $3200-4900$ & Jun-Aug & BSA 10189 (CDRI) \\
\hline Ranunculus adoxifolius Hand.-Mazz. & & + & $\mathrm{H}$ & MGM & $4600-4800$ & Jul-Aug & BSA 11002 (CDRI) \\
\hline Ranunculus bulbosus $\mathrm{L}$. & & + & H & MS & $3200-3800$ & Apr-Jun & DD \& PB 4674 (GBP) \\
\hline Ranunculus hirtellus Royle ${ }^{* *}$ & & + & $\mathrm{H}$ & MS & $4200-4900$ & May-Jul & DD \& PB 3300, 4671 (GBP); UCB 71239 (BSD)! \\
\hline Ranunculus pulchellus C.A. Mey. & + & & H & $M G, R B$ & $3200-3800$ & Jun-0ct & SKS 103048, 103062 (BSD)! \\
\hline Ranunculus trichophyllus Chaix. (Fig. 3l) & + & & $\mathrm{H}$ & MSW & $3200-4200$ & May-Aug & SKS 100395 (BSD)! \\
\hline Ranunculus trivedii Aswal \& Mehrotra & + & + & $\mathrm{H}$ & MAS & $3200-4800$ & Jun-Aug & DD \& PB 3250, 4169, 4647 (GBP); NLB 15136 (DD)! \\
\hline \multicolumn{8}{|l|}{ Rosaceae } \\
\hline Potentilla argyrophylla Wall. ex Lehm. (Fig. 4H) & + & + & H & $\mathrm{DS}, \mathrm{RB}$ & $4200-4800$ & Jun-Aug & DD \& PB 4633, 4656, 4667 (GBP); SKS 103022 (BSD)! \\
\hline Potentilla crantzii (Crantz) Beck ex Fritsch & + & & $\mathrm{H}$ & DS & $3200-4200$ & Jun-Aug & DD \& PB 3264 (GBP); SKS 107029 (BSD)! \\
\hline Potentilla multifida $\mathrm{L}$. & + & & H & $M S, R B$ & $3200-4200$ & Jun-Aug & DD \& PB 3222, 3272, 4161, 4605 (GBP); SKS 103067 (BSD)! \\
\hline Potentilla nivea $\mathrm{L}$. & & + & $\mathrm{H}$ & DST & $3200-4800$ & Jul-Aug & DD \& PB 4644, 4662, 4666 (GBP); BSA 10295 (CDRI) \\
\hline Potentilla tetrandra (Bunge) Hook.f. & & + & $H$ & DST & $4200-4800$ & May-Aug & DD \& PB 3292, 4690 (GBP) \\
\hline Sibbaldia parviflora Willd. & + & & $\mathrm{H}$ & DST & $3200-4200$ & Jun-Aug & DD \& PB 3262 (GBP) \\
\hline \multicolumn{8}{|l|}{ Rubiaceae } \\
\hline Galium acutum Edgew. & + & & H & MS & $3600-4200$ & Jun-Aug & DD \& PB 4187 (GBP) \\
\hline
\end{tabular}




\begin{tabular}{|c|c|c|c|c|c|c|c|}
\hline Botanical Name & $\begin{array}{c}\text { Chandra } \\
\text { Tal }\end{array}$ & $\begin{array}{c}\text { Suraj } \\
\text { Tal }\end{array}$ & Habit & Habitat & $\begin{array}{l}\text { Elevation } \\
\text { (m) }\end{array}$ & $\begin{array}{c}\text { Flowering \& } \\
\text { fruiting }\end{array}$ & Voucher details \\
\hline Galium aparine $\mathrm{L}$. & + & & $\mathrm{H}$ & MS & $3200-3800$ & Jun-Aug & DD \& PB 3256 (GBP); SKS 107022 (BSD)! \\
\hline \multicolumn{8}{|l|}{ Saxifragaceae } \\
\hline Bergenia ciliata (Haw.) Sternb. (Fig. 3C) & + & & H & MOR & $3800-4200$ & Mar-Jul & DD \& PB 4135 (GBP) \\
\hline Saxifraga flagellaris Willd. (Fig. 6N) & + & + & H & MST & $3600-4890$ & Jul-Aug & DD \& PB 3218, 3299, 4182, 4183 (GBP) \\
\hline Saxifraga hirculus L. & + & + & H & MST & $3800-4800$ & Jun-Sept & DD \& PB 3268, 3293, 3294 (GBP); SKS 107162 (BSD)! \\
\hline Saxifraga sibirica $\mathrm{L}$. & + & + & H & MST & $3800-4200$ & Jun-Sept & SKS 103055 (BSD)! \\
\hline Saxifraga jacquemontiana Decne. (Fig. 60) & + & + & H & MS & $3800-4900$ & Jul-Sept & DD \& PB 4158, 4640 (GBP); BSA 10986 (CDRI) \\
\hline
\end{tabular}

cylindrical. Involucral bracts linear-oblong. Corolla purple. Pappus hairs few or many, scabrid, light brown or blackish.

Threat status. Critically Endangered.

Boraginaceae

\section{Arnebia euchroma (Royle ex Benth.) I.M. Johnst. Pink Arnebia}

Material examined. INDIA - Himachal Pradesh • Rare species occurring on dry and stony slopes, LahaulSpiti district, Chandra Tal; $32^{\circ} 28.589^{\prime} \mathrm{N}, 077^{\circ} 36.951^{\prime} \mathrm{E}$; alt. 3920 m; 13.07.2017; DD \& PB 4136 (GBP).

Identification. Perennial herbs with thick purple root. Stems covered with white trichomes. Basal leaves with petiole; middle and upper cauline leaves sessile, lanceolate. Inflorescence cymes, many flowered. Corolla funnel shaped, pink or pink white. Nuts irregularly and coarsely tuberculate, greyish.

Threat status. Critically Endangered.

Crassulaceae

\section{Rhodiola heterodonta (Hook. f. \& Thomson) Boriss.} Toothed Rhodiola

Material examined. INDIA - Himachal Pradesh • Rare species occurring on rocky slopes, Lahaul-Spiti district, Suraj Tal; $32^{\circ} 45.714^{\prime} \mathrm{N}, 077^{\circ} 23.885^{\prime} \mathrm{E}$; alt. 4782 m; 19.06.2016; DD \& PB 4620 (GBP).

Identification. Perennial herbs. Rhizomes thick. Stems erect. Leaves remotely arranged, alternate, sessile, triangular-ovate. Sepals glabrous, greenish. Petals rhombic-elliptic to oblong. Follicles brown. Seeds ellipsoid, brown.

Threat status. Vulnerable.

Papaveraceae

\section{Papaver guilelmi-waldemarii (Klotzsch) Christenh. \& Byng \\ Blue Poppy}

Material examined. INDIA - Himachal Pradesh • Rare species occurring on rock crevices among boulders, Lahaul-Spiti district, Suraj Tal; $32^{\circ} 45.780^{\prime} \mathrm{N}, 077^{\circ} 23.952^{\prime} \mathrm{E}$; alt. 4780 m; 27.06.1958; MAR 5913 (BSD).

Identification. Perennial, prickly, erect herb, Stem densely bristly. Radical leaves pinnately lobed, long petioled; cauline leaves sessile. Flowers blue to purplish blue; pedicel spiny. Capsule dehiscing by 4-6 valves, 1-1.5 cm long. Seeds subreniform, brown.

Threat status. Endangered.

Polygonaceae

\section{Rheum spiciforme Royle \\ Spiked Rhubarb}

Material examined. INDIA - Himachal Pradesh • Scarcely occurring on moist sandy slopes, Lahaul-Spiti district, Chandra Tal; $32^{\circ} 29.121^{\prime} \mathrm{N}, 077^{\circ} 36.668^{\prime} \mathrm{E}$; alt. 4257 m; 21.07.2016; DD \& PB 3735; • Lahaul-Spiti district, Suraj Tal; $32^{\circ} 45.780^{\prime} \mathrm{N}, 077^{\circ} 23.952^{\prime} \mathrm{E}$; alt. $4783 \mathrm{~m}$; 11.07.2017; DD \& PB 4622 (GBP).

Identification. Perennial, short, stout herb. Leaves ovate or ovate-elliptic. Petioles of basal leaf purplish red. Panicles spiciforme. Tepals light green, elliptic or oblong-elliptic. Fruits oblong-ellipsoid. Seeds round, brown.

Threat status. Vulnerable.

Ranunculaceae

\section{Aconitum violaceum Jacquem. ex Stapf}

Violet Monkshood, Mitha Telia

Material examined. INDIA - Himachal Pradesh • Occurring in moist meadows in scattered manner, LahaulSpiti district, Chandra Tal; $32^{\circ} 28.534^{\prime} \mathrm{N}, 077^{\circ} 36.990^{\prime} \mathrm{E}$; alt. 4300 m; 08.08.1961; NCN 16764 (BSD).

Identification. Biennial herb. Roots paired, tuberculous. Stem 10-35 cm tall, erect. Leaves orbicular, lower leaves with long petiole, upper leaves sessile, lamina deeply 5-partite. Inflorescence dense raceme or corymbs. Flowers blue or violet, head ' $\mathrm{T}$ ' shaped. Carpels 5. Follicles densely hairy. Seeds obpyramidal, brown.

Threat status. Vulnerable.

\section{Discussion}

In our study of Chandra Tal and Suraj Tal of LahaulSpiti, we found these two major high-altitude wetlands to harbor various vascular plant species valuable medicinal use or of conservation value due to their threatened, endemic, or native status. For the latter species, these two wetlands play an important role in their conservation. Due its high plant diversity, the Chandra Tal area has been considered one among the core areas of Cold Desert Biosphere Reserve. Among the 188 angiosperms recorded from these wetlands, dicots and monocots were 

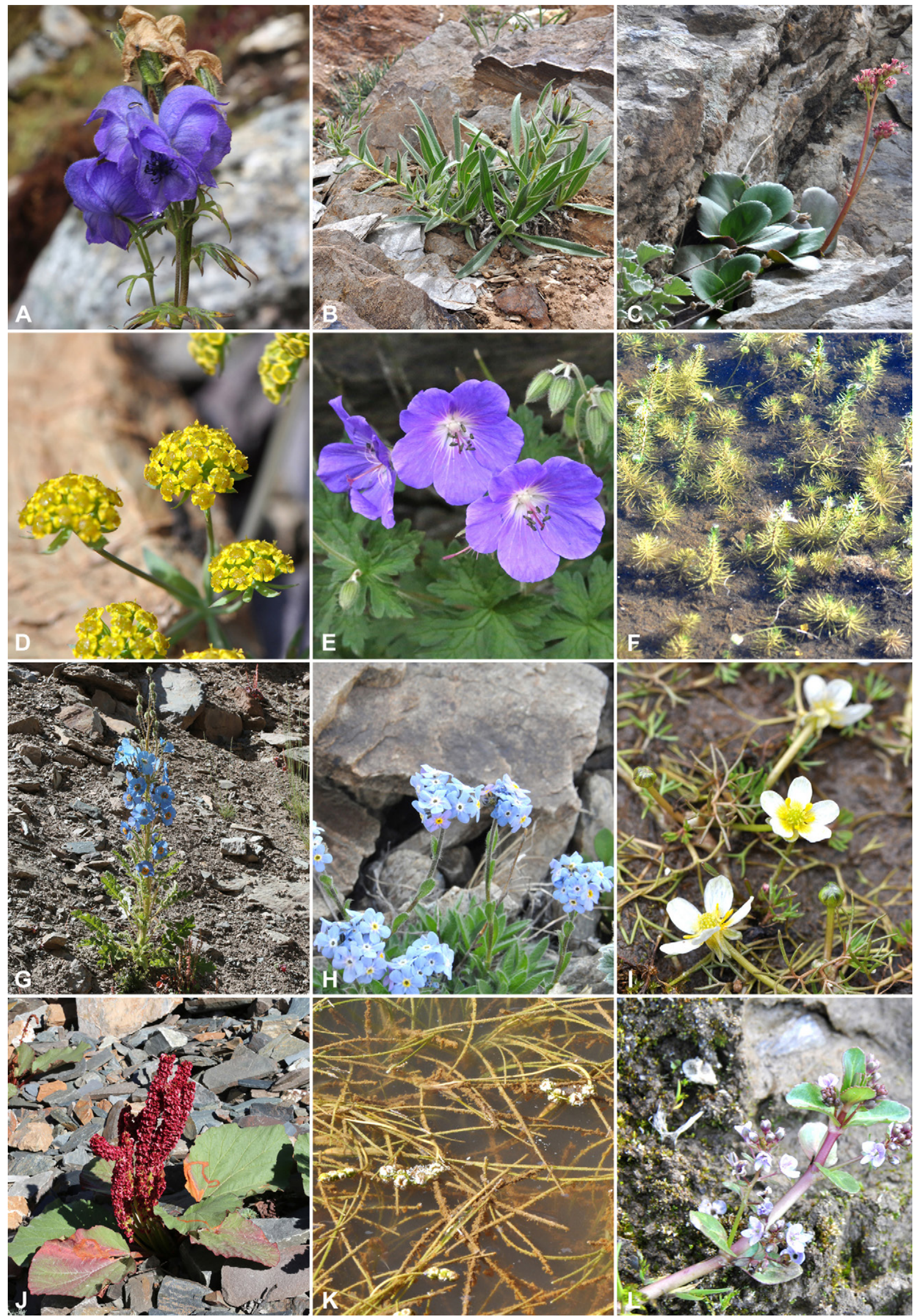

Figure 3. Vascular plants recorded in and around Chandra Tal and Suraj Tal high-altitude wetlands of Lahaul-Spiti. A. Aconitum violaceum. B. Arnebia euchroma. C. Bergenia ciliata. D. Bupleurum falcatum. E. Geranium wallichianum. F. Hippuris vulgaris. G. Papaver guilelmi-waldemarii. H. Myosotis sylvatica. I. Ranunculus trichophyllus. J. Rheum spiciforme. K. Stuckenia pectinata. L. Veronica beccabunga. 


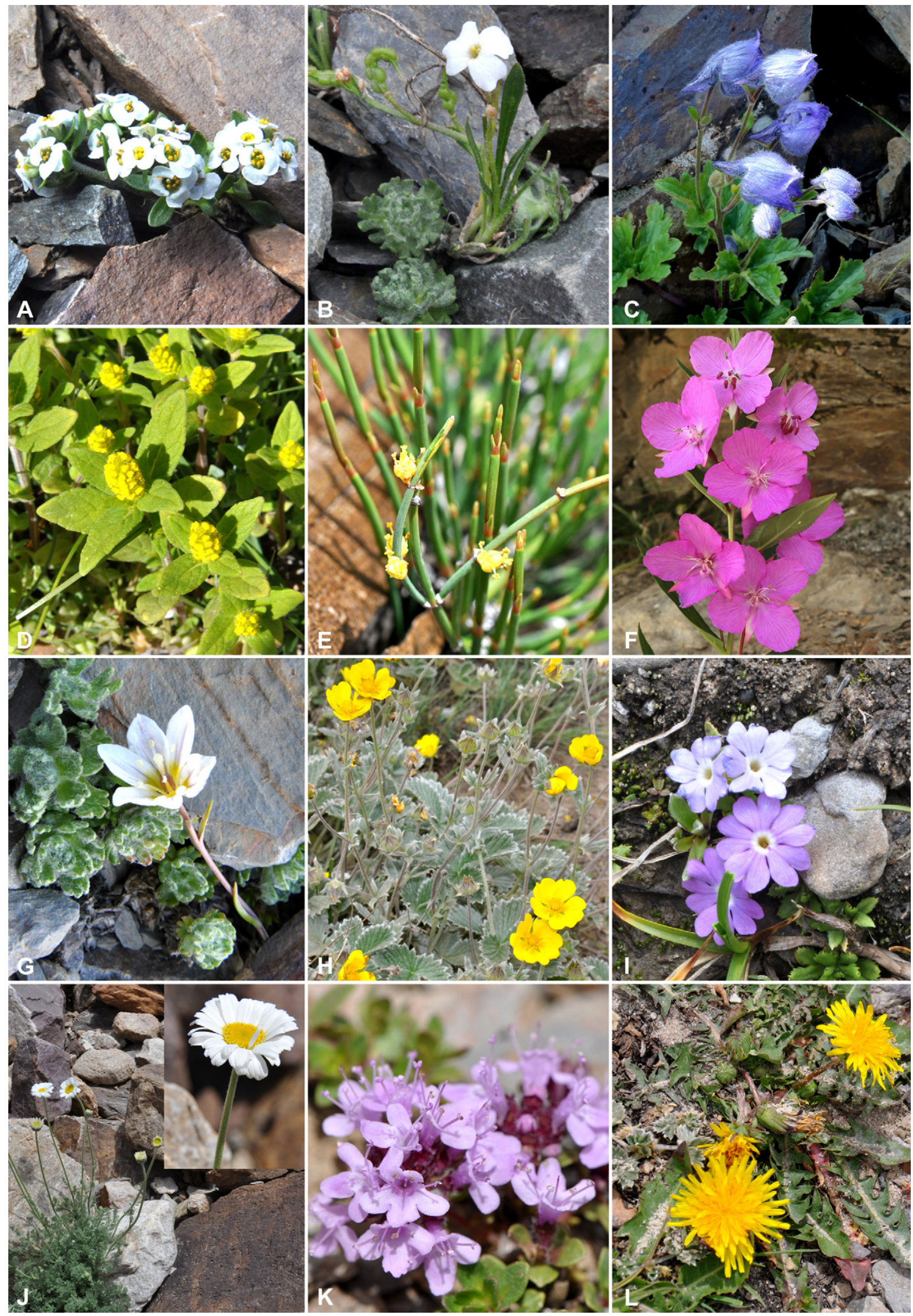

Figure 4. Vascular plants recorded in and around Chandra Tal and Suraj Tal high-altitude wetlands of Lahaul-Spiti. A. Aphragmus oxycarpus. B. Chorispora sabulosa. C. Delphinium brunonianum. D. Elsholtzia eriostachya. E. Ephedra intermedia. F. Epilobium royleanum. G. Gagea serotina. H. Potentilla argyrophylla. I. Primula minutissima. J. Richteria pyrethroides. K. Thymus linearis. L.Taraxacum officinale. 


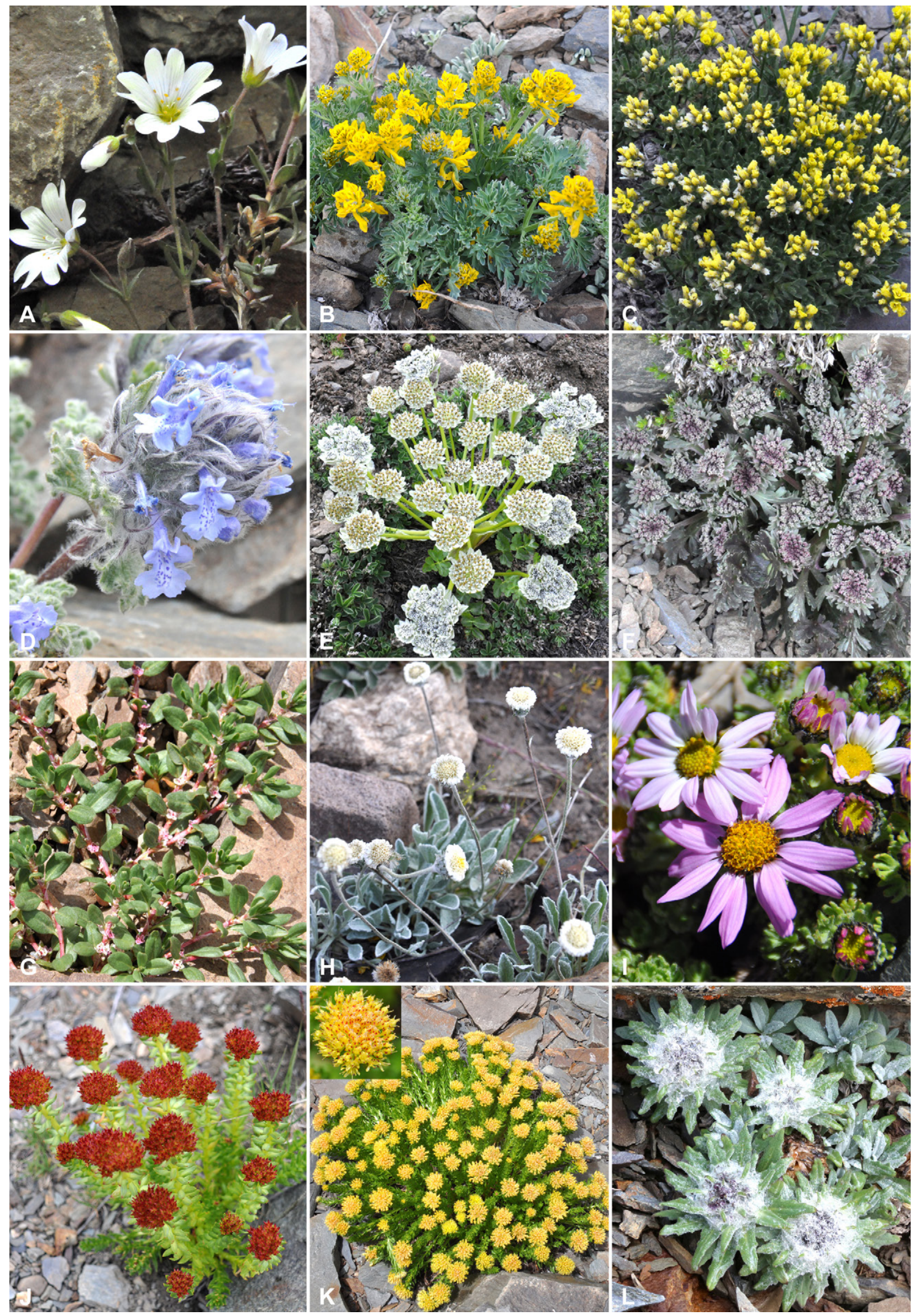

Figure 5. Vascular plants recorded in and around Chandra Tal and Suraj Tal high-altitude wetlands of Lahaul-Spiti. A. Dichodon cerastoides. B. Corydalis govaniana. C. Draba oreades. D. Nepeta longibracteata. E. Pleurospermum candollei. F. Hymenidium stellatum. G. Polygonum cognatum. H. Psychrogeton poncinsii. I. Allardia glabra. J. Rhodiola himalensis. K. Rhodiola imbricata. L. Saussurea glacialis. 


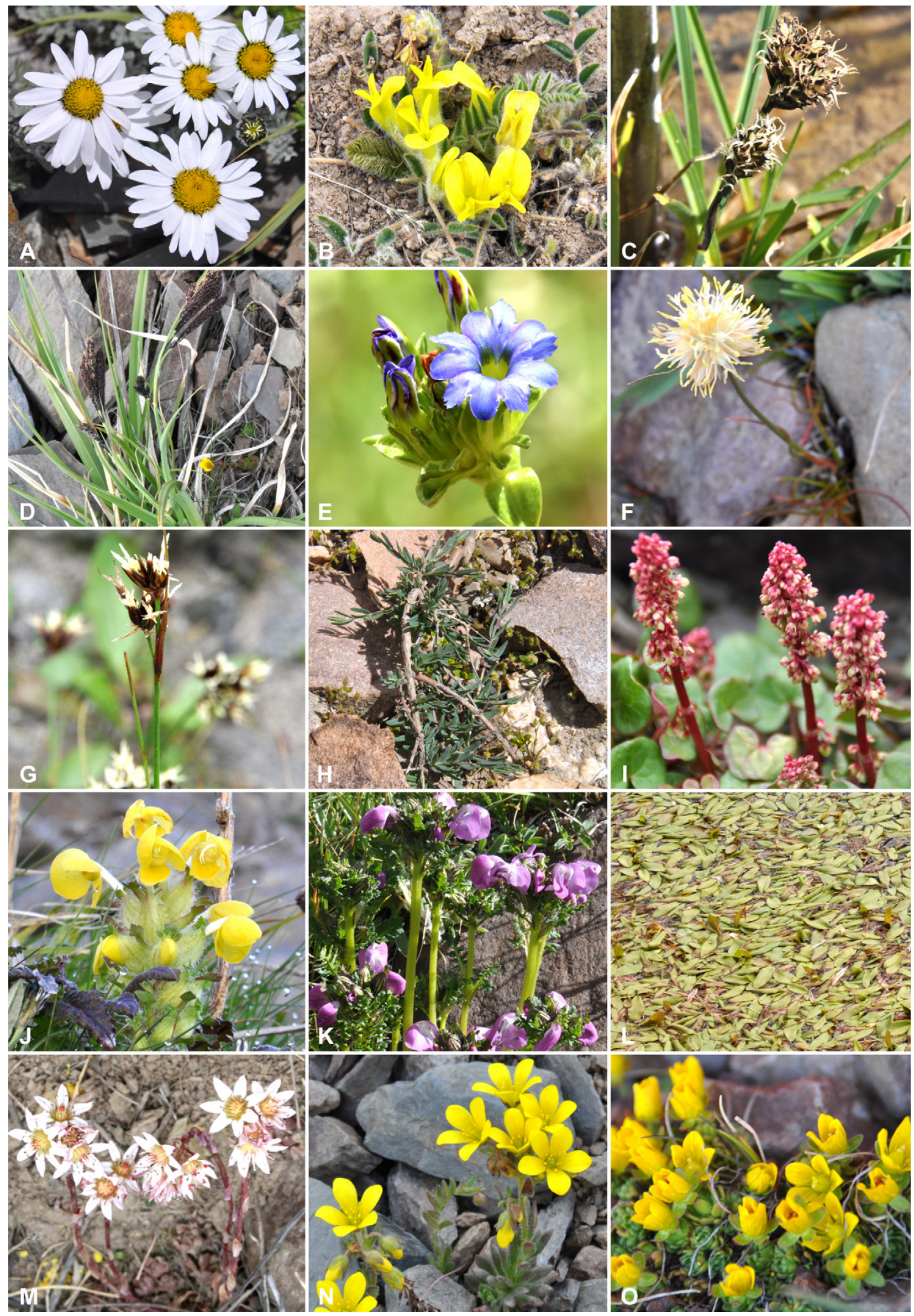

Figure 6. Vascular plants recorded in and around Chandra Tal and Suraj Tal high-altitude wetlands of Lahaul-Spiti. A. Allardia tomentosa. B. Astragalus cariensis. C. Carex maritima. D. Carex micropoda. E. Gentiana nivalis. F. Juncus allioides. G. Juncus himalensis. H. Lonicera spinosa. I. Oxyria digyna. J. Pedicularis longiflora. K. Pedicularis rhinanthoides. L. Potamogeton nodosus. M. Rosularia alpestris. N. Saxifraga flagellaris. 0. Saxifraga jacquemontiana. 
represented by 156 and 32 species, respectively. Floristic diversity was greater in Chandra Tal, with 125 species and one variety in 74 genera and 28 families, than in Suraj Tal with 98 species (in 59 genera and 23 families). Of all species recorded, 133 species (97 in Chandra Tal and 67 in Suraj Tal) were collected by us and 56 were compiled from the literature and herbaria. The two wetlands have 33 species in common. On the other hand, 93 and 65 species were exclusive in Chandra Tal and Suraj Tal, respectively. Among the 29 families recorded, over $81 \%$ of the total flora were represented by 16 dominant families. Asteraceae was the most dominant family (27 species and one variety), followed by Brassicaceae (14 species), Caryophyllaceae (13 species), Gentianaceae (13 species), Poaceae (12 species), Ranunculaceae (12 species), Cyperaceae (10 species), Fabaceae (nine species), Polygonaceae (nine species), Boraginaceae (six species), Crassulaceae (six species), Orobanchaceae (six species), Rosaceae (six species), Onagraceae, Papaveraceae, and Saxifragaceae (five species each) (Fig. 7). Campanulaceae, Caprifoliaceae, Ephedraceae, and Juncaginaceae were represented by a single species each. We found that herbs (160 species and one variety, 85.2\%) were dominant followed by grasses, rushes, and sedges (26 species, $13.8 \%$ ), and shrubs (two species, 1.1\%). We recorded the most species, 133 on moist slopes, followed by 43 species on rocky slopes, and 13 species on marshy slopes (Fig. 8). Chandra Tal had the greatest number of herbs (109 species and one variety) and grasses, rushes, and sedges (14 species) in comparison to Suraj Tal, which had 81 herb species and 15 grass, rush, and sedge species. Both wetlands had two shrubs each. Of the 188 species, six are threatened, 17 are native, and two (Eritrichium nanum and Ranunculus trivedii) are endemic to the Himalayan region.

During our field surveys, we observed high grazing pressure, heavy tourism, construction works and other anthropogenic activities, as well as harsh climatic conditions in both wetlands. We suggest that conservation of the floristic diversity in Chandra Tal and Suraj Tal requires a proper conservation strategy, an effective tourism plan, regulation to prevent overgrazing, and speciesspecific conservation plans. Strict regulation with the aim to rotate grazing areas may be especially helpful in

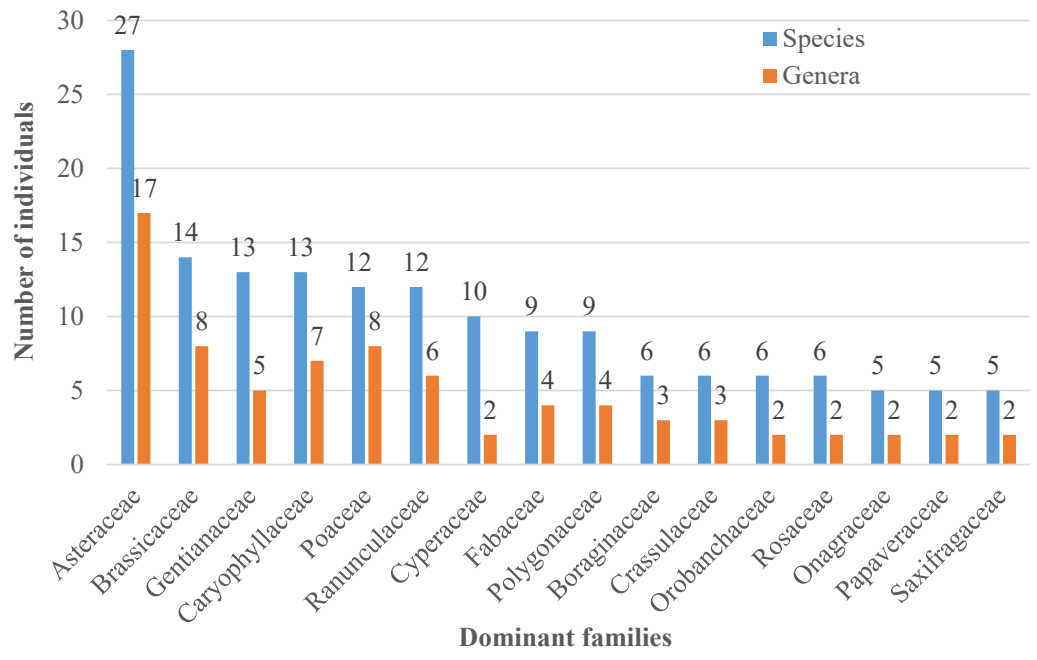

Figure 7. Dominant families with numbers of species and genera in and around Chandra Tal and Suraj Tal high-altitude wetlands of Lahaul-Spiti.

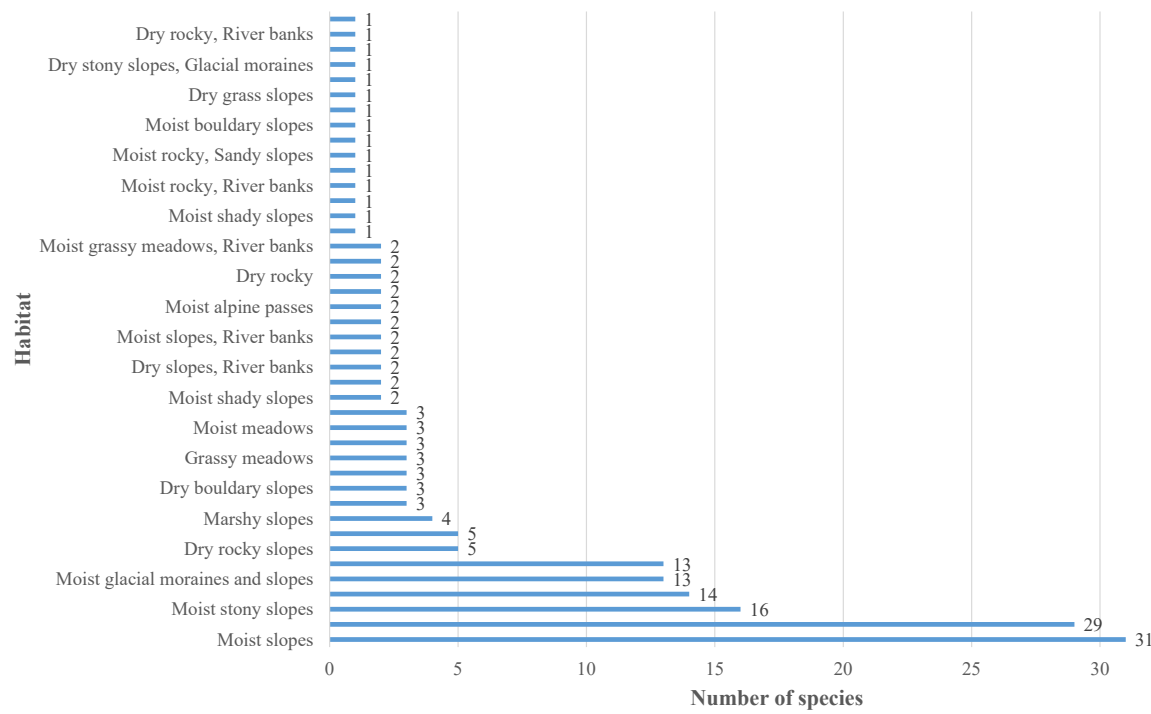

Figure 8. Distribution of species among different habitats in and around Chandra Tal and Suraj Tal high-altitude wetlands of Lahaul-Spiti. 
maintaining habitat, as would be limiting tourists during peak months. The local communities should be involved in the management and conservation of high-altitude wetlands because their economies are highly dependent on these wetlands. Their traditional knowledge should also be incorporated into the conservation work to improve the wetlands ecology and their economy. Local people management committees can help regulate tourismrelated activities, such as waste management, and also serve to guide tourists. The success of any conservation initiative will depend on the actions and cooperation of the forest department, local government administration, and the local communities.

Our findings should be of use to botanists, naturalists, conservationists, and policy makers in understanding the floristic diversity of two high-altitude wetlands in Lahaul-Spiti. Our study will also provide baseline data for further quantitative as well as qualitative investigations of valuable plants.

\section{Acknowledgements}

We are grateful to Er. Kireet Kumar (Director-inCharge) and Dr. R.S. Rawal and Dr. P.P. Dhyani (Former Directors) of the G.B. Pant National Institute of Himalayan Environment, Kosi-Katarmal, Almora or providing necessary facilities and support. We acknowledge the research support received from Ministry of Environment, Forest and Climate Change (ER Division: 14/178/2013ERS/RE). We are thankful to Principal Chief Conservator of Forest/Chief Wildlife Warden, Himachal Pradesh (WL/Research-study/WLM/1306-09) for providing necessary permission and Divisional Forest Officer, Lahaul-Spiti, for field guidance. We are thankful to $\mathrm{Mr}$. Deepak Singh Negi for field assistance and the local people of Lahaul-Spiti for field guidance, local help, and logistic support. We are also grateful to the anonymous reviewers for providing valuable comments on the manuscript.

\section{Authors' Contributions}

DD: Conceptualization, Data curation, Formal analysis; PB: Methodology, Validation; KCS: Funding acquisition, Project administration; DA: Supervision.

\section{References}

Anonymous (2009) National wetland conservation programme guidelines for conservation and management of wetlands in India. Conservation and Survey Division, Ministry of Environment and Forests, Government of India, New Delhi, India, 1- 45.

Anonymous (2010) Wetlands (Conservation and Management) Rules 2010. Gazette of India, Part II, Section 3 (ii), Government of India, 1-8.

Aswal BS, Mehrotra BN (1994) Flora of Lahaul-Spiti (a cold desert in north west Himalaya). Bishen Singh Mahendra Pal Singh, Dehradun, India, $761 \mathrm{pp}$.
Bhattacharyya UC, Uniyal BP (1982) A botanical tour to Pangi and Trilokinath in upper Chenab. Journal of Bombay Natural History Society 79: 57-78.

Brun F, Berthier E, Wagnon P, Kääb A, Treichler D (2017) A spatially resolved estimate of High Mountain Asia glacier mass balances from 2000 to 2016. Nature Geoscience 10 (9): 668-673. https:// doi.org/10.1038/ngeo2999

Chandra Sekar K, Srivastava SK (2009) Flora of the Pin Valley National Park, Himachal Pradesh. Botanical Survey of India, Kolkata, India, 296 pp.

Chandra Sekar K, Rawat B (2011) Diversity, utilization and conservation of ethnomedicinal plants in Devikund - a high altitude, sacred wetland of Indian Himalaya. Medicinal Plants 3 (2): 105-112. https://doi.org/10.5958/j.0975-4261.3.2.017

Chase MW, Christenhusz MJM, Fay MF, Byng JW, Judd WS, Soltis DE, Mabberley DJ, Sennikov AN, Soltis PS, Stevens PF (2016) An update of the Angiosperm Phylogeny Group classification for the orders and families of flowering plants: APG IV. Botanical Journal of the Linnean Society 181 (1): 1-20. https://doi.org/10.1111/ boj. 12385

Chowdhery HJ, Wadhwa BM (1984) Flora of Himachal Pradesh: Analysis. Botanical Survey of India, Calcutta, India, $860 \mathrm{pp}$.

Dhar U, Samant SS (1993) Endemic plant diversity in Indian Himalaya I. Ranunculaceae and Paeoniaceae. Journal of Biogeography 20: 659-668. https://doi.org/10.2307/2845521

IUCN (2021) The IUCN Red List of threatened species, version 20211. IUCN Red List Unit, Cambridge, UK. https://www.iucnredlist. org. Accessed on: 2021-2-9.

Jain SK, Rao RR (1977) A handbook of field and herbarium methods. Today \& Tomorrow's Printers and Publishers, New Delhi, India, $157 \mathrm{pp}$.

Joshi AC (2003) Aquatic vegetation of Lahaul. The Paleobotanist 1: $277-280$.

Kala CP (2003) Medicinal plants of Indian Trans-Himalaya: focus on Tibetan use of medicinal resources. Bishen Singh Mahendra Pal Singh, Dehradun, India, 200 pp.

Kala CP (2006) Medicinal plants of the high-altitude cold desert in India: diversity, distribution and traditional uses. The International Journal of Biodiversity Science and Management 2 (1): 43-56. https://doi.org/10.1080/17451590609618098

Kapahi BK, Sarin YK (1979) Contribution of the botany of Lahaul. Journal of Bombay Natural History Society 74: 627-639.

Koelz WN (1979) Notes on the ethnobotany of Lahul, a province of the Punjab. Quarterly Journal of Crude Drug Research 17 (1): 1-56. https://doi.org/10.3109/13880207909083272

Mehta P, Chandra Sekar K, Bhatt D, Tewari A, Bisht K, Upadhyay S, Negi VS, Soragi B (2020) Conservation and prioritization of threatened plants in Indian Himalayan Region. Biodiversity and Conservation 29 (6):1723-1745. https://doi.org/10.1007/s10531-020-01 959-x

Murti SK (2001) Flora of cold deserts of Western Himalaya. Vol. 1. (Monocotyledons). Botanical survey of India, Calcutta, India, $452 \mathrm{pp}$.

Nair NC (1964) On a botanical tour to Lahul and Spiti (Punjab Himalayas). Nelumbo 6 (2-4): 219-235.

Negi R, Verma PK, Baig S, Chandra A, Naithani HB, Verma R, Kumar A (2019) Checklist of family Poaceae in Lahaul and Spiti district (Cold Desert), Himachal Pradesh, India. Plant Science Today 6 (2): 270-274. https://doi.org/10.14719/pst.2019.6.2.500

POWO (2021) Plants of the world online. Royal Botanic Gardens, Kew, UK. http://www.plantsoftheworldonline.org/. Accessed on: 2021-11-12.

Rana MS, Samant SS (2011) Diversity, indigenous uses and conservation status of medicinal plants in Manali wildlife sanctuary, North western Himalaya. Indian Journal of Traditional Knowledge 10 (3): 439-459. https://doi.org/10.3923/ajbs.2014.151.157

Rawat GS, Adhikari BS (2005) Floristics and distribution of plant 
communities across moisture and topographic gradients in Tso Kar basin, Changthang plateau, eastern Ladakh. Arctic, Antarctic, and Alpine Research 37 (4): 539-544. https://doi.org/10.1657/1 5230430(2005)037[0539:fadopc]2.0.co;2

Rau MA (1960) On a collection of plants from Lahaul. The Bulletin of Botanical Survey of India 2 (1-2): 45-56.

Samant SS, Dhar U, Palni LMS (1998) Medicinal Plants of Indian Himalaya: Diversity Distribution Potential Values. Gyanodaya Prakashan, Nainital, India, 163 pp.

Samant SS, Palni LMS, Pandey S (2012) Cold Desert Biosphere Reserve-Trans Himalaya, India. In: Palni LMS, Rawal RS, Rai RK, Reddy SV (Eds.) Compendium on Indian biosphere reserves: progression during two decades of conservation. G. B. Pant National Institute of Himalayan Environment and Ministry of Environment, Forest and Climate Change New Delhi, India, 169-177.

Samant, Pant S, Singh M, Lal M, Singh A, Sharma A, Bhandari S (2007) Medicinal plants in Himachal Pradesh, north western Himalaya, India. The International Journal of Biodiversity Science and Management 3 (4): 234-251. https://doi.org/10. 1080/17451590709618177

Sangewar CV (2012) Remote sensing applications to study Indian glaciers. Geocarto International 2 (3): 197-206. https://doi.org/10.10 80/10106049.2011.617841

Schmidt R, Psenner R (1992) Climate changes and anthropogenic impacts as causes for $\mathrm{pH}$ fluctuations in remote high Alpine lakes. Documenta dell'Istituto Italiano di Idrobiologia 32: 31-57.
Sharma P, Samant SS (2016) Diversity of Pteridophytes in the surroundings and dam submergence areas of hydroelectric projects in Kullu district of Himachal Pradesh, Indian Himalaya. Forestry Ideas 22 (2): 127-136.

Singh A, Lal M, Samant SS (2009) Diversity, indigenous uses and conservation prioritization of medicinal plants in Lahaul valley, proposed Cold Desert Biosphere Reserve, India. International Journal of Biodiversity Science \& Management 5 (3): 132-154. https:// doi.org/10.1080/17451590903230249

Singh KN, Lal B, Singh RD, Todaria NP, Ahuja PS (2007) Species richness, distribution pattern and conservation status of higher plants in the Spiti cold desert of trans Himalaya, India. The International Journal of Biodiversity Science and Management 3 (4): 223-233. https://doi.org/10.1080/17451590709618176

Singh P, Karthigeyan K, Lakshminarasimhan P, Dash SS (2015) Endemic vascular plants of India. Botanical Survey of India, Kolkata, India, $215 \mathrm{pp}$

Srivastava SK, Shukla AN (2015) Flora of cold desert Western Himalaya, India. Vol. 2. Botanical Survey of India, Kolkata, India, 571 pp.

Ved DK, Kinhal GA, Ravikumar K, Prabhakaran V, Ghate U, Sankar V, Indresha JH (2003) Conservation assessment \& management prioritisation of selected medicinal plants of Jammu \& Kashmir, Himachal Pradesh \& Uttaranchal. Proceeding of the workshop held at Shimla during 19-24 May, 2003. Foundation for Revitalisation of Local Health Traditions, 24 pp. Unpublished report. 